\title{
The Synergy of Electrochemical Impedance Spectroscopy and Medicinal Technology
}

Edward P Randviir* and Craig E Banks

Faculty of Science and Engineering, School of Chemistry and the Environment, Division of Chemistry and Environmental Science, Manchester Metropolitan University, Chester Street, Manchester M1 5GD, Lancs, UK

\begin{abstract}
A reversed phase high performance liquid chromatographic assay coupled with UV detection [ $239 \mathrm{~nm}]$ has been developed and validated for the determination of midazolam in rat plasma samples after transdermal administration. A liquid-liquid extraction was used to extract the compound from plasma sample. The separation was performed on a Hypersil ODS C18 column using a mobile phase composed of acetonitrile and $0.1 \%$ triethylamine aqueous solution [ $52: 48, V / V$ ], pumped at a flow rate $1.0 \mathrm{~mL} \mathrm{~min}^{-1}$. The calibration curves showed good linearity with correlation coefficient higher than 0.998 for all analytes in the range $0.10-10.0 \mu \mathrm{g} \mathrm{mL}^{-1}$. Accuracy in the measurement of quality control [QC] samples was in the range $95-107 \%$ of the nominal values. The intra-day and inter-day precisions in the measurement of QC samples were less than $10 \%$ coefficient of variation. The developed method is suitable for quality control of midazolam in their mixtures and in transdermal delivery system pharmaceutical preparations. The validated assay was found to be suitable for the pharmacokinetic study of midazolam in rats with transdermal administration.
\end{abstract}

\section{Introduction}

There is no doubt as to the dramatic effect that physical and chemical technologies have had towards improving conditions for patients and employees. For example, Magnetic Resonance Imaging (MRI), founded in 1971 by Paul Lauterbur [1] is consistently used today to help diagnose joint ailments, cardiovascular problems, liver problems, and nervous system diseases [2]. The effect of MRI has been so profound that Lauterbur was awarded the Nobel Prize for medicine in 2003 [3]. There is no doubt that such technologies have had a profound effect upon the state of medical practises; MRI has improved understanding of joint and muscular pain for example, and there are many more technologies which have combined and contributed towards improving medical procedures, and effectively, reduce waiting times. X-Rays are widely used for examining bone structures, ultrasound is used for imaging unborn babies, and Enzyme Linked Immuno Sorbent Assay (ELISA) is routinely used for the detection of bacteria, viruses, and antibodies [4].

However, this does not mean that all diseases are objectively quantifiable; nor does it mean that these techniques are cheap; a standard MRI machine will cost a hospital upwards of $£ 500,000$, and high running costs mean that unnecessary usage cost the health services greatly. Many techniques are not quick either; ELISA, for example, requires samples to be taken to a lab, which increases the chance of sample contamination, and take at least 24 hours to complete [5]. These latter points are currently being addressed by researchers, and in particular, electrochemical methods have been pin-pointed by some as an answer to portability and cost issues invoked by disease measurement procedures such as ELISA [6,7]. Indeed, point-of-care procedures have been researched which certainly have the capability of portability, when used in conjunction with screen-printed electrodes [7-9] which are small devices (typically $3-4 \mathrm{~cm}$ in length) which consist of printed carbon layers, and have the ability to specifically detect elements, ions, molecules, proteins, and even viruses and disease. In order to do this, a voltage must be applied across such a printed circuit (Figure 1), which contains a working electrode (where the target is detected), an auxiliary electrode (to provide the current), and a reference electrode (to reference the potential changes to). The current produced at the working electrode is measured and interpreted by cheap, portable electrochemical apparatus. The equipment used to measure the analytical signal from which information on the target can be obtained can be easily applied to the measurements of compounds in urine, blood, saliva, or serum samples using a technique known as Electrochemical Impedance Spectroscopy (EIS).

EIS is a technique which has been used for over a century, [10] yet its practical application in terms of bioanalytical chemistry has only been realised within the past 20 years. EIS probes an electrochemical cell with a small-range sinusoidal voltage and a DC potential at the working electrode, and measures the sinusoidal current produced at the working electrode surface as a result of a combination of Faradaic (electron transfer) and non-Faradaic (physical changes) processes. The ratio of the sinusoidal voltage and current is expressed as the impedance of the cell, but impedance is a non-ideal quantity, thus doubling the voltage does not necessarily mean a double in the current. In a standard EIS experiment, a range offrequencies are applied to the system in order to more accurately validate any chemical and physical processes which take place. More information regarding the technique can be found in Barsoukov's or Bard's texts [11,12].

Simply put, EIS can be applied in medicinal technologies by observing non-Faradaic phenomena. Viruses and bacteria exhibit molecular structures that are too big to probe using charge transfer reactions, therefore physical changes have to be monitored to

${ }^{*}$ Corresponding author: Edward P Randviir, Faculty of Science and Engineering School of Chemistry and the Environment, Division of Chemistry and Environmental Science, Manchester Metropolitan University, Chester Street, Manchester M1 5GD, Lancs, UK, Tel: 01612471196; Fax: 01612476831; E-mail: EdRandviir@ gmail.com, c.banks@mmu.ac.uk

Received November 27, 2014; Accepted January 13, 2015; Published January 16,2015

Citation: Randviir EP, Banks CE (2015) The Synergy of Electrochemical Impedance Spectroscopy and Medicinal Technology. Pharm Anal Acta 6: 336 doi:10.4172/2153-2435.1000336

Copyright: @ 2015 Randviir EP, et al. This is an open-access article distributed under the terms of the Creative Commons Attribution License, which permits unrestricted use, distribution, and reproduction in any medium, provided the original author and source are credited. 


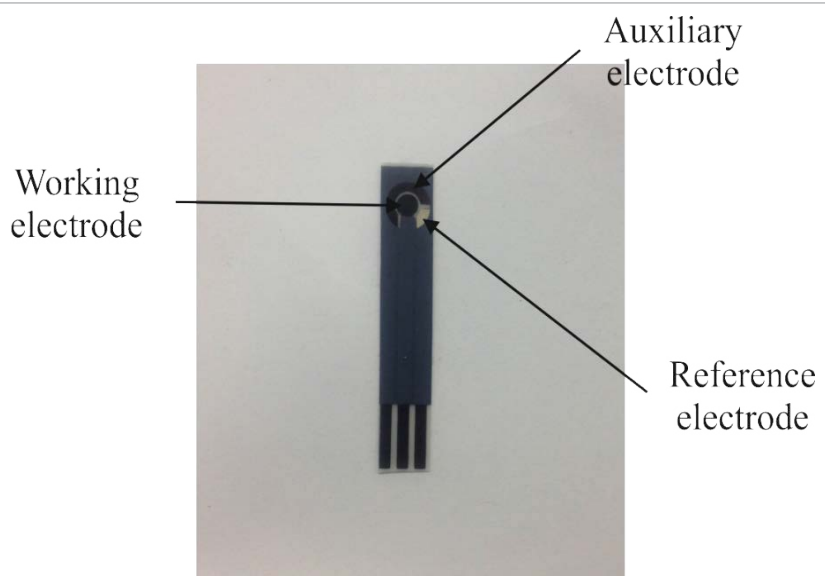

Figure 1: Schematic of a carbon screen-printed electrode.

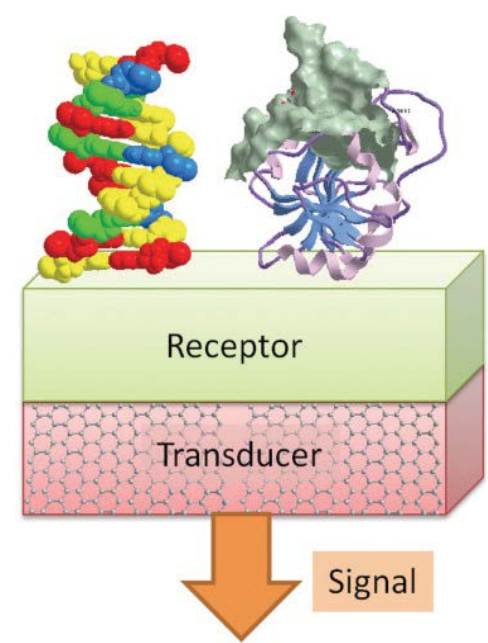

Figure 2: A generic working electrode for a medical application. A composite support is applied to the working electrode, which acts as an electrochemical transducer by amplifying any physicochemical changes as a transient current to the electrochemical equipment. The receptor layer consists, normally, of a protein, biomolecule, or DNA sequence, which specifically targets the disease that is being probed. Figure reprinted courtesy of Elsevier [17].

analyse samples; which can, quite paradoxically, be probed using electrochemical methods! A generic procedure for the measurement of a disease using EIS is depicted in (Figure 2). To create a working electrode capable of detecting a disease, one must functionalize the surface in a manner in which it will only interact with the specific disease in question. Normally, a working electrode surface will be modified with a composite material that has the capability of transducing physicochemical changes, such as graphene or carbon nanotubes. Bare electrodes such as glassy carbon would of course be suitable, but the amplification derived from other carbon materials/ composites renders results that are more accurate. The interactive part of the electrode is immobilised upon the transduction layer, either by self-absorbing by immersion in a solution of the desired coating, or by pipetting an aliquot of the material upon the electrode surface. With some minor finishing, the electrode will then be ready to detect the desired target, which will be suspended in a bodily fluid such as blood, saliva, urine, or serum.

There are numerous examples of such reports within the scientific literature. Some highly cited examples of such technologies can determine the presence of E. Coli, [13] lysozyme, [14] and Salmonella [15]. The latter example reports a detection time of less than six minutes, which is remarkable, considering the current method (ELISA) would take over 24 hours for the analytical procedure to be complete. Such techniques also could potentially detect disease levels (i.e. detect microbe levels quantitatively) for applications in the food standards industry.

Today, the focus is towards branching out these technologies towards more diseases, and hopefully in the future, clinical procedures will incorporate EIS as a method to help medical practises in the diagnosis of illnesses. This would provide a quick and cheap method to objectively assess a patient's illness, while reducing waiting times due to rapid diagnosis and treatment. The field needs to develop methods which can be utilized in one-shot, disposable ways, such as through the use of printed circuits, $[16,17]$ in order to make this technique a commercially viable option for disease diagnosis. That said, it may not be too long until somebody finds Alzheimer's disease or a sexually transmitted disease being diagnosed in the doctor's surgery. Electroanalytical chemistry is emerging and growing by the day, and it is coming to you too.

\section{References}

1. Lauterbur PC (1989) Image formation by induced local interactions. Examples employing nuclear magnetic resonance. 1973. ClinOrthopRelatRes : 3-6.

2. NHS UK, Accessed November 2014.

3. Nobelprize.org, Accessed November 2014.

4. Suzuki K, Sawada T, Murakami A, Matsui T, Tohma S, et al. (2003) High diagnostic performance of ELISA detection of antibodies to citrullinated antigens in rheumatoid arthritis, Scandinavian Journal of Rheumatology 32:197-204.

5. Leng SX, McElhaney JE, Walston JD, Xie D, Fedarko NS, et al. (2008) Elisa And Multiplex Technologies For Cytokine Measurement In Inflammation And Aging Research, J Gerontol A BiolSci Med Sci63: 879-84.

6. Randviir EP, Banks CE (2013) Analytical methods for quantifying creatinine within biological media, Sensors and Actuators B Chemical 183: 239-52.

7. Banks CE,Randviir EP (2014) Detection of creatinine: technologies for point-ofcare determination of glomerular filtration. Bioanalysis 6: 109-111.

8. Randviir EP,Kampouris DK, Banks CE (2013) An improved electrochemical creatinine detection method via a Jaffe-based procedure. Analyst 138: 65656572.

9. Randviir EP, Metters JP, Stainton J, Banks CE (2013) Electrochemica impedance spectroscopy versus cyclic voltammetry for the electroanalytica sensing of capsaicin utilising screen printed carbon nanotube electrodes, Analyst 138: 2970-2981.

10. Randviir EP, Banks CE (2013) Electrochemical impedance spectroscopy: an overview of bioanalytical applications, Analytical Methods 5:1098-1115.

11. Barsoukov E, Macdonald JR(2005) Impedance spectroscopy: Theory, experiment, and applications (2nd Edn) Wiley.

12. Bard AJ, Faulkner L (2001) Electrochemical methods: Fundamentals and applications (2nd Edn) John Wiley \& Sons.

13. Maalouf R, Fournier-Wirth C, Coste v, Chebib H, Saïkali Y, et al.(2007) LabelFree Detection of Bacteria by Electrochemical Impedance Spectroscopy: Comparison to Surface Plasmon Resonance, Analytical Chemistry 794879 794886.

14. Rodriguez MC,Kawde AN, Wang J (2005) Aptamer biosensor for label-free impedance spectroscopy detection of proteins based on recognition-induced switching of the surface charge. ChemCommun (Camb) : 4267-4269.

15. Nandakumar V, La Belle JT, Reed J, Shah M, Cochran D, et al. (2008) A methodology for rapid detection of Salmonella typhimurium using label-free electrochemical impedance spectroscopy. BiosensBioelectron 24: 1045-1048.

16. Metters JP,Kadara RO, Banks CE (2011) New directions in screen printed electroanalytical sensors: an overview of recent developments. Analyst 136: 1067-1076.

17. Pumera M (2011)Graphenein biosensing, Materials Today: 14308-14315 